which the nation is able to use its scientists and technologists. But both Mr. Greenwood's survey and that of Mr. McCrensky deserve to be studied by the Minister of Education and the Lord President of the Council, for they point unmistakably to the essential factors which will determine the success or failure of efforts in Britain to increase the supply of scientists and technologists to a level commensurate with the nation's needs, and to ensure that the maximum use is made of the men and women thus trained.

\section{CONCEPTS OF NUCLEAR PHYSICS}

\section{Nuclear Structure}

By Leonard Eisenbud and Prof. Eugene P. Wigner. (Investigations in Physics, Vol. 8.) Pp. viii +128. (Princeton, N.J. : Princeton University Press; London : Oxford University Press, 1958.) 25s. net.

\section{T}

HERE are surprisingly few up-to-date textbooks of theoretical nuclear physics which may be recommended to the final-year honours student or to the postgraduate beginner. Many books have been written containing a wealth of detail toth inessential and confusing to the beginner; several which treat particular topics at some length but fail to give any sense of balance; only one or two of the calibre of this one, and these are mostly out of date owing to the speed at which nuclear physics has grown. In the space of a little more than one hundred pages the authors present a clear and balanced account of the whole of low-energy nuclear physics. As one would expect of Prof. E. P. Wigner and Dr. L. Eisenbud, the book is entirely theoretical in its approach, although essentially non-mathematical. That is to say, the authors have dispensed with the usual long-winded accounts, so common in elementary text-books, of the process of acquiring the data on which the interpretation is based. There are some nuclear physicists who will frown at this even to-day : yet even they would not expect to describe the workings of an ammeter during a discourse on the conductivity of metals, or the properties of spectroscopes while expounding the electronic structure of atoms. The techniques of nuclear physics, both experimental and mathematical, can be found elsewhere: here one will find the concepts.

The first three chapters are descriptive, giving the general features of nuclei, their energy systematics and ground-state properties. As an illustration of how up to date the book is, one notes that the values quoted for nuclear radii are those recently obtained by Hofstadter using the scattering of highenergy electrons. The fourth chapter gives a correspondingly descriptive account of the various types of nuclear reactions. At the end of this chapter occurs about the only reference to practical matters, in the delightfully succinct sentence: "Nuclear reactions can be used to produce radioactive elements". The fifth chapter gives a brief account of the properties of the two-body system and of nuclear forces. Here again one finds a modern picture, with Levy's potential, complete with hard core, appearing in the most unembarrassing fashion. The three following chapters are concerned with the various models which have been put forward to try to understand nuclear properties. Only the essential assumptions and most significant results of each model are discussed, and the relations between them. There follow two chapters on the theory of nuclear reactions, the first of which gives a beautiful and almost painless account of the powerful $R$-matrix theory (or rather of its essential concepts, for it is only the details which are gruesome and these are omitted, in keeping with the rest of the book), the second an account of strip. ping reactions and Coulomb excitations. Resonance formulæ, reduced widths, radioactivity, complex potentials, giant resonances-they are all included within a very few pages, without fuss, and yet, somehow, with sufficient explanation to make one feel one really understands it all. The final two chapters deal with beta-decay and with electromagnetic transitions. Here, unfortunately, the book is already obsolete : scalar and tensor reign supreme and parity goes inviolate. A most valuable addition is the list of carefully selected references, which are commented upon by the authors so that an inexperienced reader can easily find his way to the vast modern literature.

The critical reader may certainly find some faults. There seem to be one or two misprints, and it is a pity that the word 'Fermi' has been introduced as a synonym for 'barn'. Although the usage is historically correct, the Fermi is now almost fully established as the nuclear unit of length. One may wonder why the $L S$ - and $j j$-coupling models are presented in such contrasting tones: most people to-day would regard them as extremes of a very narrow band of colours. But these are quibbles. It is an excellent little book, warmly to be recommended to anyone who wishes to obtain an authoritative and balanced view of the concepts of nuclear physics. Even the price is a $\begin{array}{ll}\text { welcome surprise. } & \text { B. H. FLOwERS }\end{array}$

\section{FLUID SYSTEMS IN CHEMICAL ENGINEERING}

\section{Chemical Engineering Practice}

Edited by Herbert W. Cremer and Trefor Davies. Vol. 5 : Fluid Systems I. Pp. vi $+695+$ xxiv. (London : Butterworths Scientific Publications; New York: Academic Press, Inc., 1958.) 95s.; 13.30 dollars.

THERE is probably no aspect of chemical en. gineering that has received greater attention. and which so clearly indicates the special nature of this technology, than that of distillation. In the petroleum industry the installed capacity for distillation is greater than that for any other operation, and the modern petrochemical industry as well as the conventional organic chemical industry are wide users of distillation equipment. A large section of this volume is taken up with an account of the techniques of, and equipment for, distillation, and for this reason alone this work will receive much atten. tion. The allied operation of liquid - liquid extraction is also covered, so that we have here an exposition of two of the really important chemical engineering operations which have been widely developed by chemical engineers. There are other important sections which will prove of considerable value not only to the professional chemical engineer but also to that wider body of engineers whose work brings them to chemical engineering from time to time. The main topies discussed are transportation of liquids and 